\title{
Mixed mode ductile fracture using the strain energy density criterion
}

\author{
C.L. CHOW ${ }^{(1)}$ and JILIN XU ${ }^{(2)}$ \\ (1) Department of Mechanical Engineering, University of Hong Kong, Hong Kong \\ (2) Institute of Mechanics, Academia Sinica, Beijing, China
}

(Received May 31, 1984; in revised form November 8, 1984)

\begin{abstract}
An investigation of extending the application of the strain energy density criterion to mixed mode ductile fracture is described. Thin plates embedded with a center crack inclined at $\beta=30$ and 60 degrees are analysed. An Euclerian finite element formulation is used for the large elastic-plastic flow problem incorporating isotropically hardening Prandtl-Reuss material and incremental deformation.

An alternative criterion based on the maximum dilatational energy for determining crack initiation angle in ductile fracture is proposed. Based on this criterion, the predicted fracture loads for the cracked plates of $\beta=30$ and 60 degrees are compared satisfactorily with the measurements.
\end{abstract}

\section{Introduction}

The concepts of linear elastic fracture mechanics (LEFM) in terms of the stress intensity factor $K_{\mathrm{IC}}$ or its equivalent $G_{\mathrm{C}}$ are now accepted by practising engineers for quantitative assessments of the onset of failure in structures containing defects. The general acceptance of LEFM is however limited strictly to truly brittle materials and those, such as high strength steels, which fracture with only small amounts of irreversible deformation localized at the tip of defects. Unfortunately for most commonly used structural and pressure vessel materials under normal working environments, this limitation becomes unrealistic as fracture of these materials is often preceded by an extensive gross plastic deformation under service loadings.

In the last sixteen years, there has been an intensive research effort in the development of a failure criterion for post-yield fracture as summarized adequately by Latzko [1]. No attempt is made here to provide a complete review on the subject of post-yield fracture mechanics and interested readers may refer to the cited reference for information. It may suffice however to mention that among many different post-yield fracture criteria so far proposed, the concepts of $J$-integral [2,3] crack-opening displacement (COD) $[4,5]$ and strain energy density ( $S$-theory) [6,7] have received their respective popularity. The dependence of specimen geometry and mode of loading as well as their inconsistent effects on the crack growth have cast considerable doubts on the validity of COD and $J$-integral concepts as fundamentally sound fracture control of parameters [8-11]. The strain energy density criterion has on the other hand been shown to be capable of characterizing not only brittle fracture [12] but also ductile fracture by Sih and Madenci [7,13]. They demonstrated the application of the strain energy density criterion to solve mode I ductile fracture of thin center cracked plates undergoing gross plastic deformation.

This paper describes an investigation of extending the application of the strain energy 
density criterion to mixed mode fracture of thin plates stressed beyond general yielding. This is achieved by analysing a central crack of length $2 \mathrm{a}$ inclined at an angle $\beta$ in finite length and width thin plates subjected to uniaxial tension $\sigma_{0}$ as shown in Fig. 1. The variations of angle $\beta$ considered include 30 and 60 degrees. The nonlinear elastic-plastic finite element analysis is performed incorporating both nonlinear material and geometrical behaviors for predicting fracture loads at the onset of crack initiation. The predicted results are then compared with those determined experimentally in order to examine the validity and accuracy of the strain energy density criterion for mixed mode ductile fracture studies.

\section{Theoretical analysis}

According to the strain energy density criterion due to Sih $[12,13]$, the location of maximum yielding is postulated to occur when the strain energy density reaches its maximum value, $(\mathrm{d} W / \mathrm{d} V)_{\max }$, whereas fracture initiation is associated with minimum strain energy density, $(\mathrm{d} W / \mathrm{d} V)_{\min }$. In addition, for failure due to gross plastic deformation, crack initiation and growth are postulated to be governed when $(\mathrm{d} W / \mathrm{d} V)_{\max }$ and $(\mathrm{d} W / \mathrm{d} V)_{\min }$ reach their respective critical values. The critical strain energy density function $(\mathrm{d} W / \mathrm{d} V)_{\mathrm{c}}$ may be determined from the area underneath the true stress-strain diagram or using the following expression

$$
\left(\frac{\mathrm{d} W}{\mathrm{~d} V}\right)_{\mathrm{c}}=\int_{0}^{\epsilon_{u}} \sigma \mathrm{d} \epsilon
$$

where $W$ is stored strain energy, $V$ is volume element of the material used, $\epsilon_{\mathrm{u}}$ is the ultimate strain, $\sigma$ is true stress and $\epsilon$ is true strain measured under monotonic loading from a standard test specimen. For material failure under gross plastic yielding, the material resistance to fracture is decreased by the degree of irreversible deformation, say,

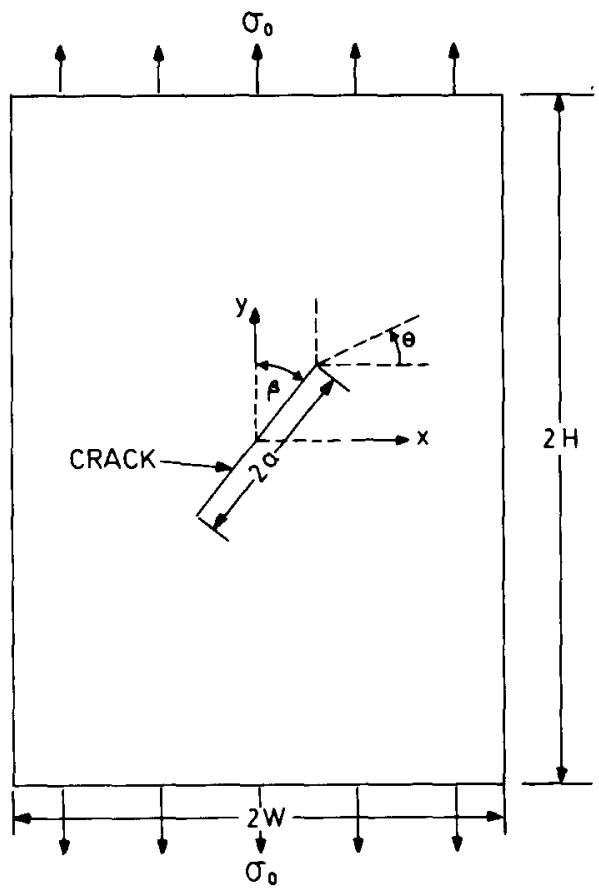

Figure 1. An inclined crack. 
point $\mathrm{p}$ beyond the material yield point on the stress-strain curve. This amount of material degradation, $(\mathrm{d} W / \mathrm{d} V)_{\mathrm{p}}$, must then be taken into consideration for evaluating the remaining ability of the material to resist fracture as

$$
\left(\frac{\mathrm{d} W}{\mathrm{~d} V}\right)_{\mathrm{c}}^{*}=\left(\frac{\mathrm{d} W}{\mathrm{~d} V}\right)_{\mathrm{c}}-\left(\frac{\mathrm{d} W}{\mathrm{~d} V}\right)_{\mathrm{p}}
$$

The above modification is only necessary for fracture within a plastic enclave but for brittle fracture, the absorbed specific energy $(\mathrm{d} W / \mathrm{d} V)_{\mathrm{c}}=(\mathrm{d} W / \mathrm{d} V)_{\mathrm{c}}^{*}$ as the contribution from the term, $(\mathrm{d} W / \mathrm{d} V)_{\mathrm{p}}$, is considered negligible. Based on the strain energy density criterion, crack initiation is assumed to occur when the strain energy density $(\mathrm{d} W / \mathrm{d} V)$ in an element around the crack tip reaches its critical material resistance value $(\mathrm{d} W / \mathrm{d} V)_{c}^{*}$.

The strain energy density distribution $(\mathrm{d} W / \mathrm{d} V)$ may be evaluated from the finite element analysis for a cracked plate as

$$
\frac{\mathrm{d} W}{\mathrm{~d} V}=\int \sigma_{i j} \mathrm{~d} \epsilon_{i j}
$$

where $\sigma_{i j}$ denote stresses and $\epsilon_{i j}$, strains. From physical considerations, excessive change in shape or distortion in a volume element is associated with yielding while excessive change in volume or dilatation is associated with fracture. The strain energy density may also be divided into two parts. The term involving the dilatational energy and distortion energy are respectively given by (see Appendix)

$$
\begin{aligned}
& \left(\frac{\mathrm{d} W}{\mathrm{~d} V}\right)_{\mathrm{v}}=\frac{1}{3} \int \sigma_{l l} \mathrm{~d} \epsilon_{k k} \\
& \left(\frac{\mathrm{d} W}{\mathrm{~d} V}\right)_{\mathrm{d}}=\int \sigma_{i j}^{\prime} \mathrm{d} \epsilon_{i j}^{\prime}
\end{aligned}
$$

such that

$$
\left(\frac{\mathrm{d} W}{\mathrm{~d} V}\right)=\left(\frac{\mathrm{d} W}{\mathrm{~d} V}\right)_{\mathrm{v}}+\left(\frac{\mathrm{d} W}{\mathrm{~d} V}\right)_{\mathrm{d}}
$$

Because of the development of intensive plastic deformation around the crack tip, a finite element analysis incorporating both geometrical and material nonlinearities was adopted. This was accomplished with an Eulerian finite element formulation based on Hill's variational principal for incremental deformation for isotropically hardening Prandtl-Reuss materials [14]. The analysis included the use of tangent modulus incremental plasticity and a first order self-correcting subroutine [15]. The formulation of rate equilibrium at any large deformation is given by the following form of virtual work equation [16]

$$
\int_{V}\left[\tau_{i j}^{*} \delta D_{i j}-\frac{1}{2} \sigma_{i j} \delta\left(2 D_{i k} D_{k j}-v_{k, i} v_{k, j}\right)\right] \mathrm{d} V=\int_{s} \dot{f}_{i} \delta v_{i} \mathrm{~d} S+\int_{V} \dot{b}_{i} \delta v_{i} \mathrm{~d} V
$$

where $V$ is element volume, $\tau_{i j}^{*}$ is Jaumann co-rotational rate of Kirchhoff stress, and $\mathrm{D}_{\mathrm{ij}}$ is deformation rate. $\{\mathrm{v}\}$ is the vectors of velocity components, $v_{k, i}$ is $\partial v_{k} / \partial x_{i}$ for the position vector $\boldsymbol{X}$ of a material in the current state, $\dot{f}_{i}$ is force vector intensity rate and $\dot{b}$ is body force intensity rate. The velocity vector $\{v\}$ and the deformation rate $\{D\}$ may be expressed as

$$
\{v\}=[N]\{\dot{\psi}\}
$$

and

$$
\{D\}=[B]\{\dot{\psi}\}
$$

where $\{\dot{\psi}\}$ is the vector of rates of nodal degrees of freedom. An Eulerian finite element 
rate equilibrium equation based on the virtual work formulation of (6) may be derived as

$$
[K]\{\dot{\psi}\}=\{\dot{P}\}
$$

where

$$
\{\dot{P}\}=\int_{V}[N]^{T}\{\dot{b}\} \mathrm{d} V+\int_{S}[N]^{T}\{\dot{f}\} \mathrm{d} S
$$

and

$$
[K]=\left[K_{\mathrm{ep}}\right]+\left[K_{\mathrm{s}}\right]
$$

for which

$$
\begin{aligned}
& {\left[K_{\mathrm{ep}}\right]=\int_{V}[B]^{T}[C][B] \mathrm{d} V} \\
& {\left[K_{\mathrm{s}}\right]=\int_{V}\left[N_{k}\right]_{, i}^{T} \boldsymbol{o}_{i j}\left[N_{k}\right]_{, j}-2\left[B_{k i}\right]^{T} \sigma_{i j}\left[B_{k j}\right] \mathrm{d} V .}
\end{aligned}
$$

It can be observed from the above formulation that $\left[K_{\text {ep }}\right]$ is the conventional small displacement stiffness matrix and $\left[K_{\mathrm{s}}\right]$ is associated with the geometric nonlinear term.

\section{Numerical results}

The finite element analysis was performed to predict fracture loads of two thin flat specimens, each embedded with a central crack inclined at $\beta=30^{\circ}$ and $60^{\circ}$ (see Fig. 1). Due to the antisymmetric nature of the mixed mode problem the entire plate was descretized as shown in Fig. 2, while the finite element representation of the crack tip is described in Fig. 3. For the typical example of the cracked plate with a slant angle of $30^{\circ}$, a total of 52 elements and 314 nodal points was employed.

The materials chosen for the present investigation are aluminium alloys namely

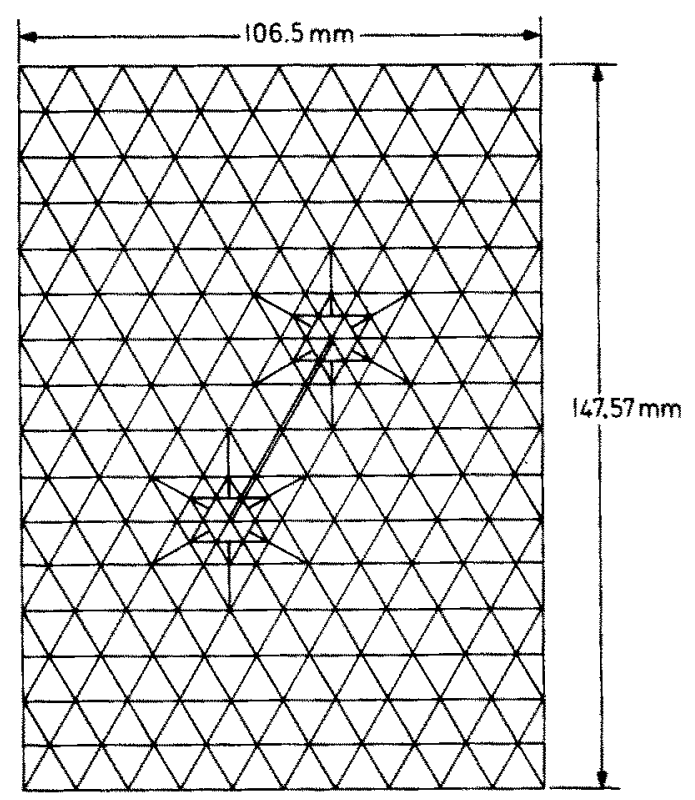

Figure 2. Finite element representation of mixed mode fracture. 


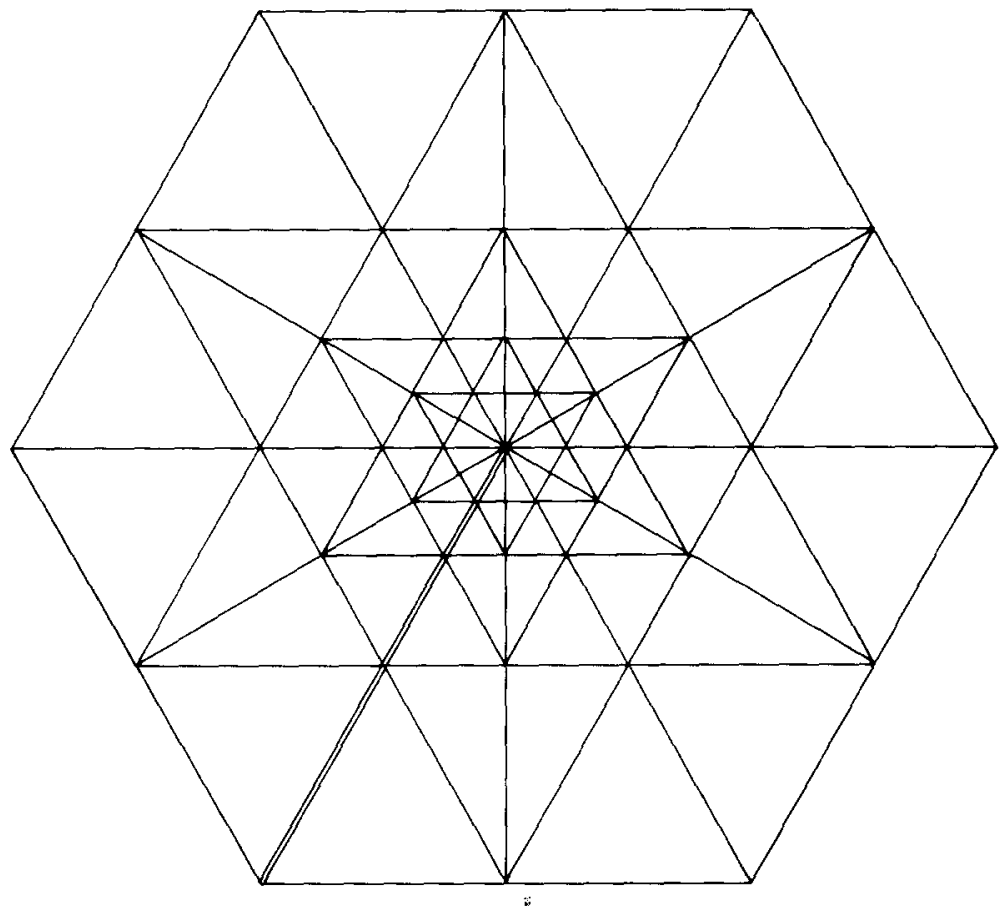

Figure 3. Cracked tip finite element discretization.

LY12-CZ and LY12-CS. The mechanical properties of the materials were for the first instance measured and summarized in Table 1. The coefficient $\alpha$ and the strain hardening exponent $n$ shown in the table were evaluated based on the following power hardening law:

$$
\epsilon=\frac{\sigma}{E}+\alpha\left[\left(\frac{\sigma}{\sigma_{\mathrm{ys}}}\right)^{n}-1\right],
$$

where $E$ is Young's modulus, $\sigma$ is true stress, $\epsilon$ is true strain, and $\sigma_{\mathrm{ys}}$ is the yield stress.

Equation (10) is used to describe material behavior of a standard tensile specimen under monotonically increasing load. The multi-axial stress distribution computed from a cracked thin plate is related to the uniaxial tensile data via the equivalent stress $\sigma_{\mathrm{e}}$ as

$$
\sigma_{e}=\left(\frac{3}{2} \sigma_{i j}^{\prime} \sigma_{i j}^{\prime}\right)^{1 / 2},
$$

where $\sigma_{i j}^{\prime}$ is the stress deviator tensor. The resistance to crack growth, $(\mathrm{d} W / \mathrm{d} V)_{\mathrm{c}}^{*}$, is evaluated using (1), (2) and (10). The measured true stress-strain curve and the material resistance to crack growth versus true stress for the test material, LY12-CZ, are described in Fig. 4. The dimensions chosen for the cracked plates embedded with an inclined crack are $147.6 \mathrm{~mm}$ in height (or $2 \mathrm{H}$ ) and $106.5 \mathrm{~mm}$ in width (or $2 \mathrm{~W}$ ).

Figure 5 depicts the computed strain energy density contours near a crack of $42.6 \mathrm{~mm}$

Table 1. Mechanical properties

\begin{tabular}{lllllll}
\hline Material & $\begin{array}{l}\text { Young's } \\
\text { modulus } E, \mathrm{GPa}\end{array}$ & $\begin{array}{l}\text { Yield } \\
\text { stress } \sigma_{\mathrm{ys}}, \mathrm{MPa}\end{array}$ & $\begin{array}{l}\text { Ultimate } \\
\text { stress } \sigma_{\mathrm{u}}, \mathrm{MPa}\end{array}$ & $\begin{array}{l}\text { Maximum } \\
\text { elongation } \epsilon_{\mathrm{f}}, \mathrm{mm}\end{array}$ & $\alpha$ & $\mathrm{n}$ \\
\hline LY12 - CZ & 69.48 & 320 & 510 & 0.169 & 0.012 & 5.3 \\
LY12 - CS & 69.48 & 320 & 489 & 0.134 & 0.013 & 5.0 \\
\hline
\end{tabular}



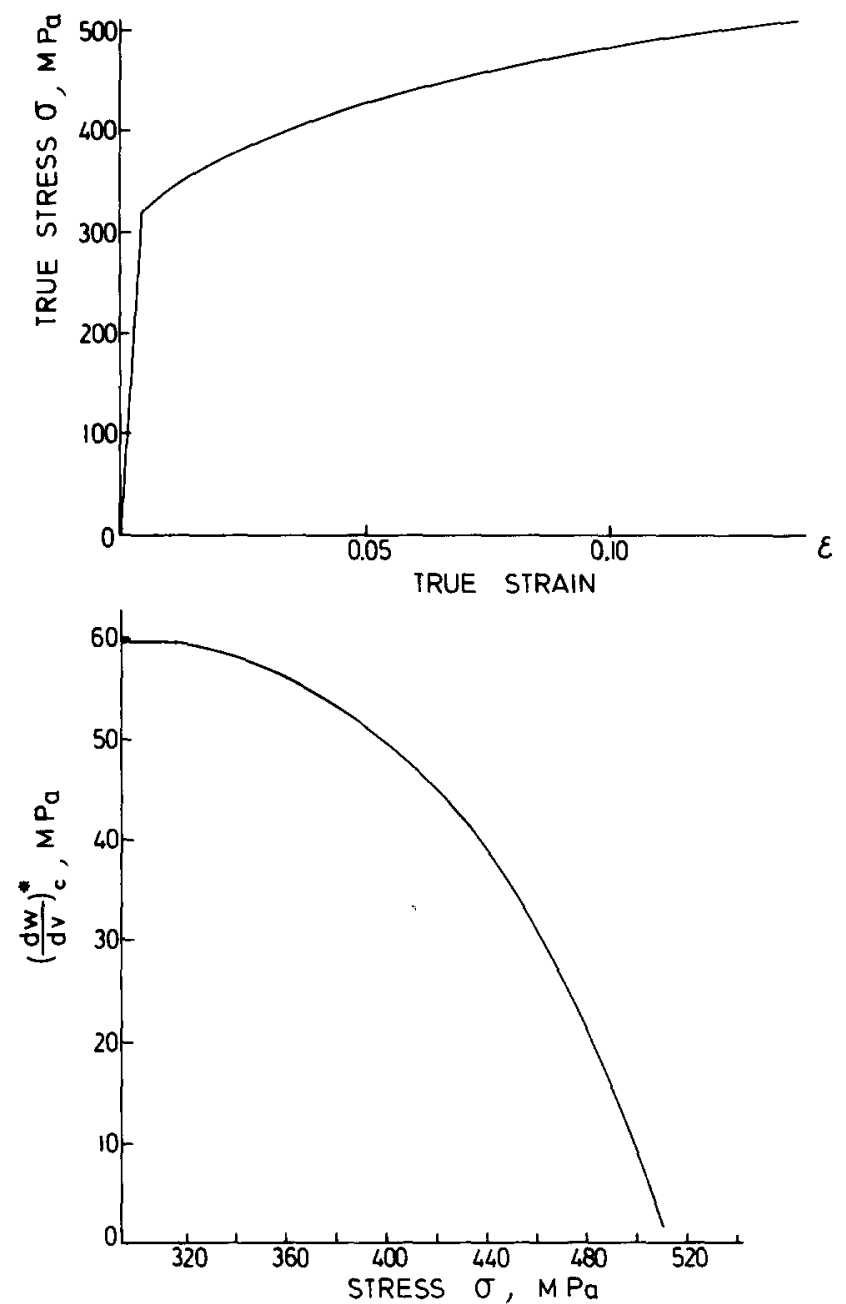

Figure 4. Resistance to crack growth for LY12-CZ aluminium alloy.

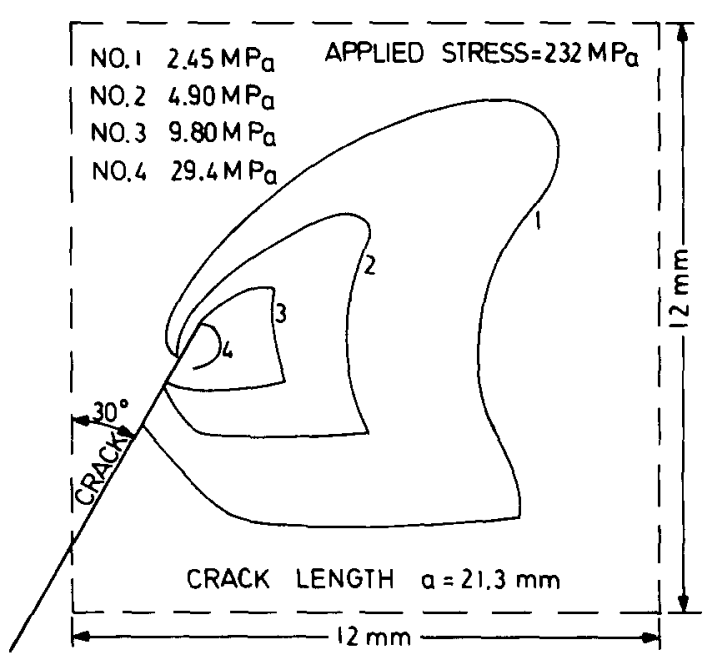

Figure 5. Strain energy density contours for $\beta=30$ degree. 


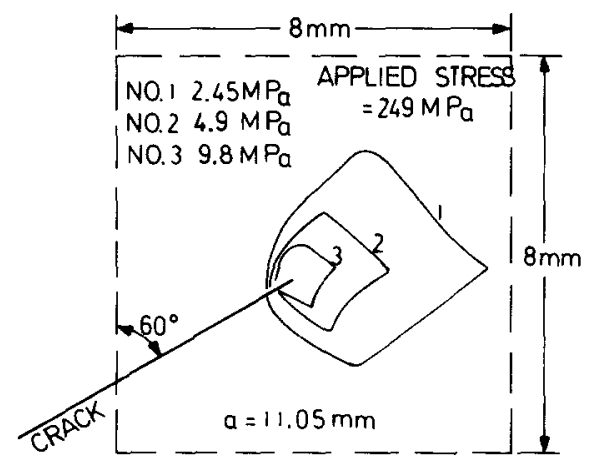

Figure 6. Strain energy density contours for $\beta=60$ degree.

(or 2a) in length slanted at $\beta=30$ degree and Fig. 6, $22.1 \mathrm{~mm}$ in length at $\beta=60$ degree. Because of the anti-symmetrical nature of the mixed mode problem, the strain energy contours near one end of the crack tips are shown.

The magnitudes of the strain energy density contours shown in Figs. 5 and 6 were developed at the applied stresses of $232 \mathrm{MPa}$ and $249 \mathrm{MPa}$ respectively causing gross plastic deformation near the crack tips. Based on the strain energy density criterion, it is assumed that excessive development of dilatation energy, $(\mathrm{d} W / \mathrm{d} V)_{\mathrm{v}}$, is associated with fracture $[7,12]$. The assumption holds irrespective of whether fracture initiation takes place in elastic or plastic portion of the material. In addition, the criterion also postulates that the location of maximum yielding is assumed to coincide with maximum strain energy density $(\mathrm{d} W / \mathrm{d} V)_{\max }$ and of fracture initiation with minimum strain energy density $(\mathrm{d} W / \mathrm{d} V)_{\min }$. In order to test the validity of the above hypotheses, the angle of crack initiation of thin plates embedded with a crack angle $\beta=90$ degree undergoing gross plastic deformation was first analysed. This example was chosen because at the slant angle, the cracked plate is in mode I condition and the angle of crack initiation is known to be zero (see Fig. 1). The analysis was based on the computed finite element results reported elsewhere by the authors [17]. The same aluminium alloy, LY12-CZ, was adopted for that investigation. The height and width of the specimen chosen were $180 \mathrm{~mm}$ and 108 $\mathrm{mm}$ respectively. A center crack of $11.55 \mathrm{~mm}$ in length was embedded in the rectangular plate.

The computed strain energy density in terms of $(\mathrm{d} W / \mathrm{d} V)$ and $(\mathrm{d} W / \mathrm{d} V)_{\mathrm{y}}$ against the angle of rotation at the crack tip are shown in Fig. 7. It can be observed from the figure that the locations of $(\mathrm{d} W / \mathrm{d} V)_{\min }$ and maximum $(\mathrm{d} W / \mathrm{d} V)_{\mathrm{v}}$ do not coincide with one another. The site where $(\mathrm{d} W / \mathrm{d} V)_{\min }$ is located at $\theta=180$ degree whereas the maximum

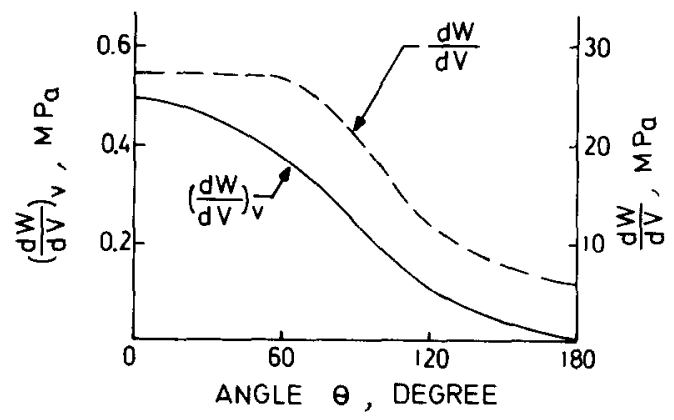

Figure 7. $(\mathrm{d} W / \mathrm{d} V)$ and $(\mathrm{d} W / \mathrm{d} V)_{\mathrm{v}}$ versus $\theta$ for $\beta=90$ degree. 


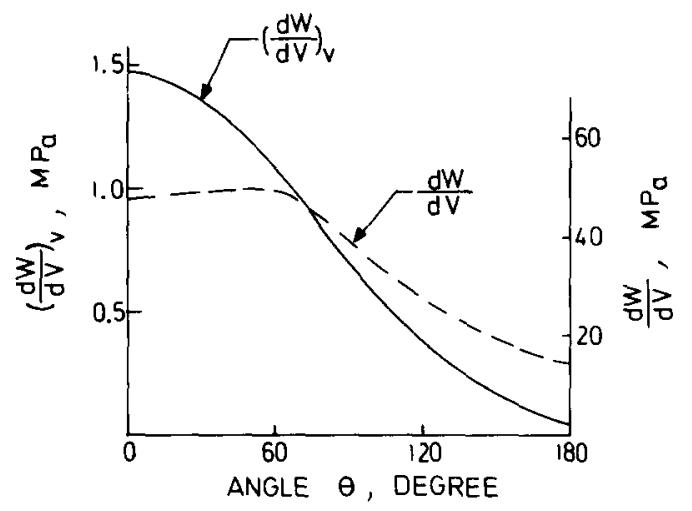

Figure 8. (dW/dV) and ( $\mathrm{d} W / \mathrm{d} V)_{\text {v }}$ versus $\theta$ for $\beta=90$ degree (from Refs. [7] and [13]).

$(\mathrm{d} W / \mathrm{d} V)_{\mathrm{v}}$ is located at $\theta=0$ degree. Since the crack initiation angle for the cracked plate is known at $\theta=0$ degree, it can be concluded that the criterion of angle of crack initiation based on the maximum $(\mathrm{d} W / \mathrm{d} V)_{v}$ should be adopted rather than the use of $(\mathrm{d} W / \mathrm{d} V)_{\min }$.

This conclusion may be further verified by a similar analysis due to Sih and Madenci $[7,12]$ for a center cracked plate with $\beta=90$ degree. Their results in terms of $(\mathrm{d} W / \mathrm{d} V)$ and $(\mathrm{d} W / \mathrm{d} V)_{\mathrm{v}}$ are replotted against angle of rotation $\theta$ at the crack tip, Fig. 8. It can be observed from the figure that the angles of rotation, $\theta$, where $(\mathrm{d} W / \mathrm{d} V)_{\min }$ and the maximum $(\mathrm{d} W / \mathrm{d} V)_{\mathrm{v}}$ are located, again differ; i.e. the angles $\theta$ for $(\mathrm{d} W / \mathrm{d} V)_{\min }$ and maximum $(\mathrm{d} W / \mathrm{d} V)_{\mathrm{v}}$ are respectively 180 and 0 degrees. The criterion of crack initiation angle described in the present investigation for ductile fracture is therefore based on the maximum of $(\mathrm{d} W / \mathrm{d} V)_{\mathrm{v}}$ rather than the minimum of $(\mathrm{d} W / \mathrm{d} V)$. The main advantage of this criterion for ductile crack initiation is that it is in agreement with the experience gained in the past for predicting angle of crack initiation for brittle materials using the criterion of $(\mathrm{d} W / \mathrm{d} V)_{\min }$ [18]. Since for brittle materials, dilatation energy $(\mathrm{d} W / \mathrm{d} V)_{\mathrm{v}}$, is predominant over distortion energy $(\mathrm{d} W / \mathrm{d} V)_{\mathrm{d}}$, the equivalent condition of $(\mathrm{d} W / \mathrm{d} V)_{\min }$ holds for the materials because the strain energy density $(\mathrm{d} W / \mathrm{d} V)$ reaches its minimum when $(\mathrm{d} W / \mathrm{d} v)_{v}$ attains its maximum value.

The computed values of $(\mathrm{d} W / \mathrm{d} V)$ and $(\mathrm{d} W / \mathrm{d} V)_{\mathrm{v}}$ for cracked plates with $\beta=30$ and 60 degrees are shown respectively in Figs. 9 and 10. It can be observed from the figures that the crack initiation angles do not coincide when the conditions that $(\mathrm{d} W / \mathrm{d} V)_{\min }$ and

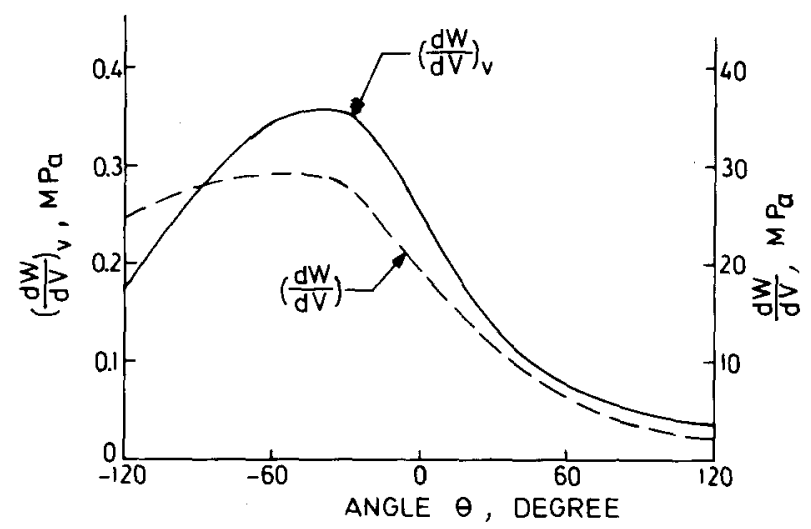

Figure 9. Variation of $(\mathrm{d} W / \mathrm{d} V)_{\vee}$ and $(\mathrm{d} W / \mathrm{d} v)$ with angle $\theta$ at $\sigma=269 \mathrm{MPa}$ and $r=0.66 \mathrm{~mm}$ for $\beta=30$ degree. 


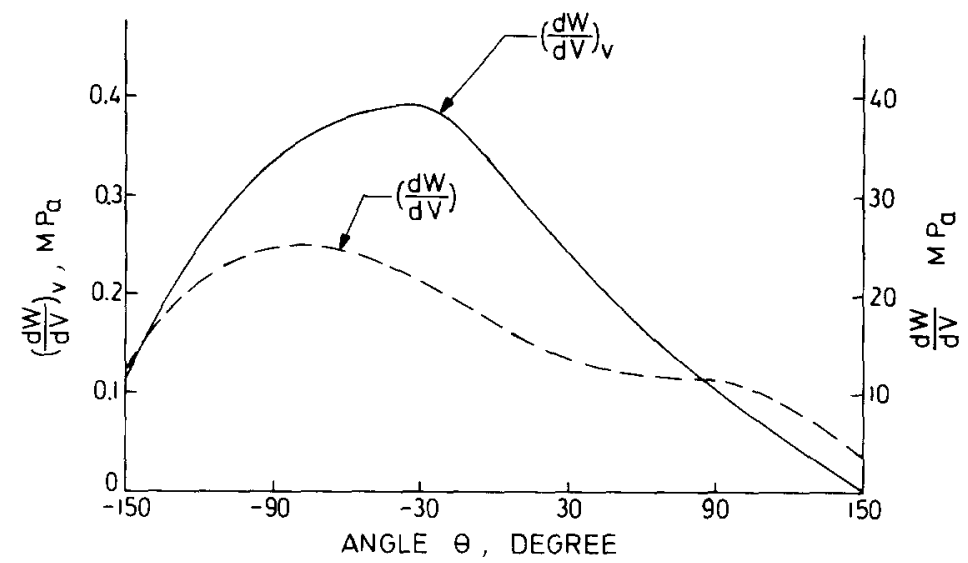

Figure 10. Variation of $(\mathrm{d} W / \mathrm{d} V)_{v}$ and $(\mathrm{d} W / \mathrm{d} V)$ with angular $\theta$ at $\sigma=249 \mathrm{MPa}$ and $r=0.35 \mathrm{~mm}$ for $\beta=60$ degree.

maximum $(\mathrm{d} W / \mathrm{d} V)_{v}$ are individually satisfied. For both the inclined angles, the maximum $(\mathrm{d} W / \mathrm{d} V)_{\mathrm{v}}$ is located at $\theta=-30$ degree where the crack initiation is predicted to take place.

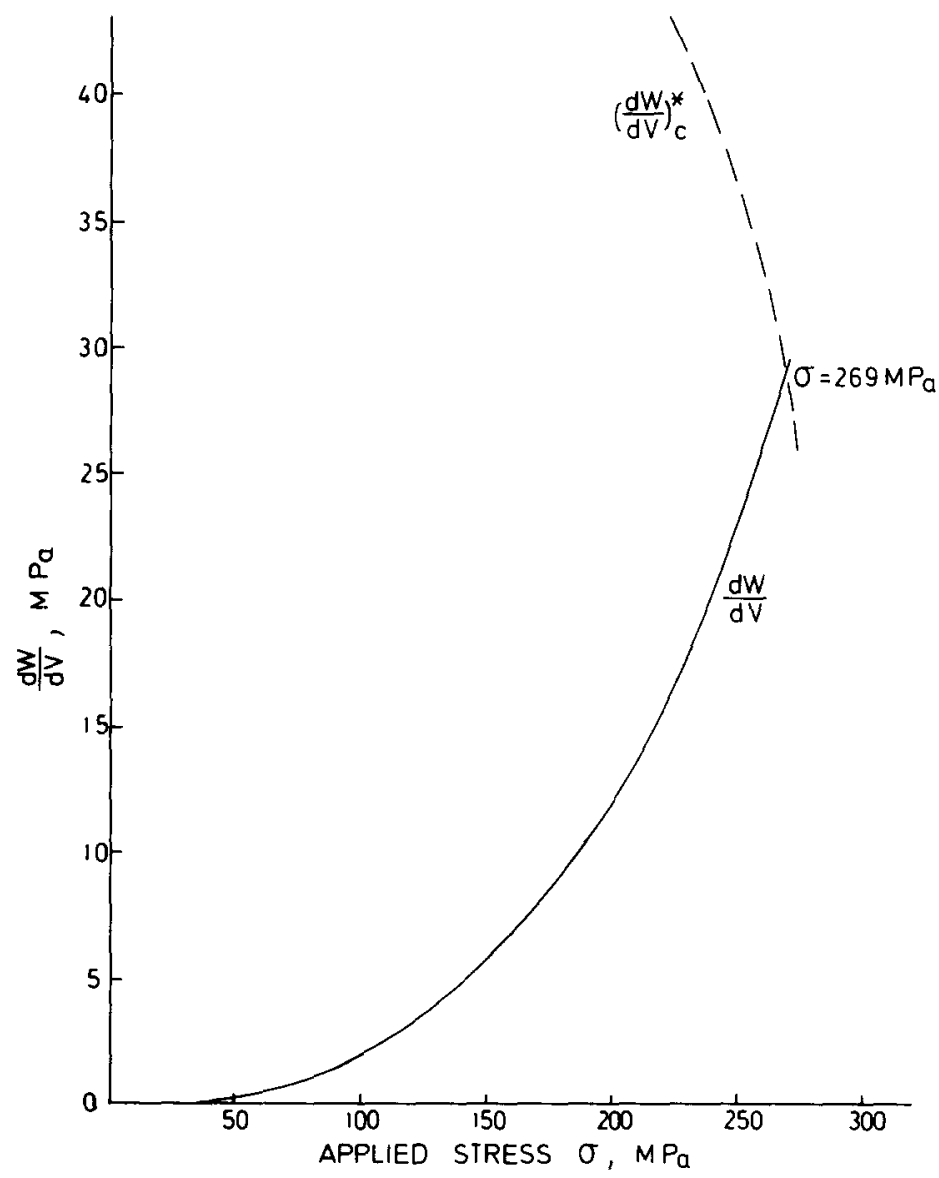

Figure 11. Strain energy density at the direction of $\theta=-30^{\circ}$ and $r=0.66 \mathrm{~mm}$ for $\beta=30$ degree. 
After the determination of angle of crack initiation, the fracture load causing the onset of crack initiation is subsequently analyzed. Based on the strain energy density criterion for ductile fracture, crack initiation is assumed to occur when the strain energy density, $(\mathrm{d} W / \mathrm{d} V)$, along the direction of the predicted angle ahead of the crack tip reaches the material resistance parameter, $(\mathrm{d} W / \mathrm{d} V)_{\mathrm{c}}^{*}$ [12]. This condition is satisfied along the direction $\theta=-30$ degree at the radial distance $r=0.66 \mathrm{~mm}$ ahead of the crack tip when the center-cracked plate of $\beta=30$ degree is loaded up to a fracture load of $269 \mathrm{MPa}$, Fig. 11. The fracture load of $249 \mathrm{MPa}$ is predicted as shown in Fig. 12 when $(\mathrm{d} W / \mathrm{d} V)=$ $(\mathrm{d} W / \mathrm{d} V)_{\mathrm{c}}^{*}$ at $r=0.35 \mathrm{~mm}$ for the cracked plate of $\beta=60$ degree.

\section{Experiments and discussion of results}

In order to check the validity and accuracy of the mixed mode analysis for ductile fracture, a series of experiments was performed to measure fracturing loads [19]. The test specimens were loaded in a tensile testing machine and the load was applied incrementally to about $70 \%$ of their respective critical loads. The critical load refers to the load at which further crack growth leads to global instability. Continuous crack growth was recorded using a travelling microscope of 80 magnifications. Because of the use of thin plate thickness of less than $1.5 \mathrm{~mm}$, the difference in the crack length measurement at the plate surface and at the mid-plane across the plate thickness is considered negligible. The microscope chosen was able to read up to $0.01 \mathrm{~mm}$. No experimental difficulties were experienced in recording the threshold of crack initiation and crack growth with the microscope.

The measured and predicted fracture loads are summarized in Table 2 together with

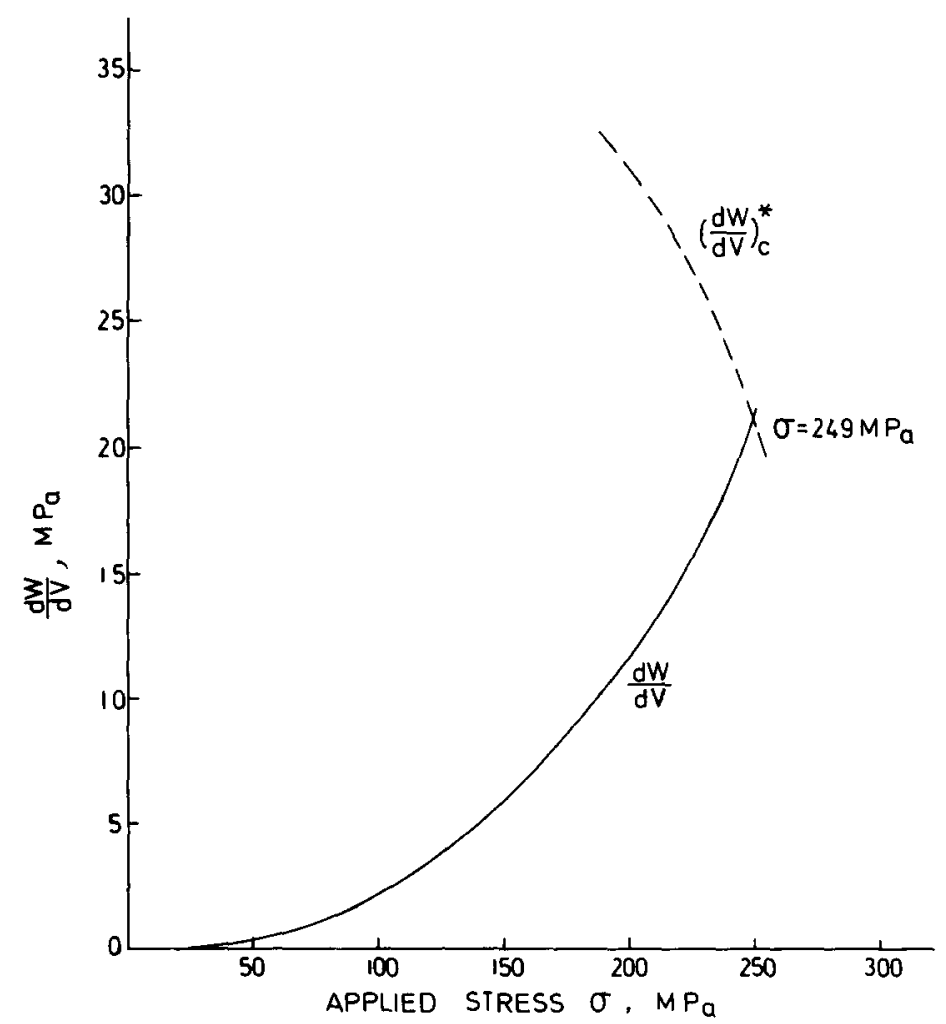

Figure 12. Strain energy density at the direction of $\theta=-30^{\circ}$ and $r=0.35 \mathrm{~mm}$ for $\beta=60$ degree. 
Table 2. Fracture loads

\begin{tabular}{lllllll}
\hline Slant angle $\beta$, degree & \multicolumn{2}{l}{ Fracture load, MPa } & & \multicolumn{2}{l}{ Crack increment $\Delta a, \mathrm{~mm}$} & Fracture load difference (\%) \\
\cline { 2 - 3 } & Analysis & Measurement & & Analysis & Measurement & \\
\hline 30 & 269 & 241 & 0.66 & 0.10 & 10.4 \\
60 & 249 & 263 & 0.35 & 0.15 & 5.3 \\
\hline
\end{tabular}

their corresponding crack increments. For the cracked plate of $\beta=30$ degree, the percentage error of 10.5 between the measured and predicted fracture loads can be observed from the table. This error is twice as much as the percentage error in the predicted and measured fracture loads for the cracked plate of $\beta=60$ degree. The difference in the percentage error for the cracked plates is attributed mainly to the relatively coarse elements chosen for the $\beta=30$ degree plate at $\Delta a=0.66 \mathrm{~mm}$ as compared with those for the $\beta=60$ degree plate at $\Delta a=0.35 \mathrm{~mm}$ (see Table 2). Lower predicted fracture load is expected if smaller crack increment than $\Delta a=0.66 \mathrm{~mm}$ was chosen, thus improving overall accuracy of the solution.

\section{Conclusions}

The application of the strain energy density criterion is extended to solve mixed mode fracture in ductile materials undergoing gross yielding. Two angled cracks each inclined at $\beta=30$ and 60 degrees in rectangular plates are considered. The predicted fracture loads are found to be satisfactory as compared with those obtained experimentally, confirming the validity of the strain energy density approach to mixed mode ductile fracture.

An alternative criterion for predicting the angle of crack initiation is proposed. This is based on the hypothesis that a crack initiates at the location where the dilatation energy reaches its maximum. This criterion is applicable to crack initiation not only within a plastic enclave but also in an elastic medium. The criterion is also shown to be equivalent to the criterion of $(\mathrm{d} W / \mathrm{d} V)_{\min }$ due to Sih in the elastic case.

It is worth emphasizing that the conclusions derived above are based on cracked thin plates subjected to uniform loading. Further investigations are in progress to examine the effects of different materials, geometries, thicknesses, and modes of loading for verifying general acceptability of the strain energy approach to ductile fracture.

\section{References}

[1] D.G.H. Latzko, Post-Yield Fracture Mechanics, Applied Science Publishers Ltd., London (1979).

[2] J.R. Rice, ASME Journal of Applied Mechanics 35 (1968) 379-386.

[3] J.W. Hutchinson, ASME Journal of Applied Mechanics 50 (1983) 1042-1051.

[4] A.A. Wells, British Welding Research Journal (1963) 563-370.

[5] F.M. Burdekin and D.E.W. Stone, Journal of Strain Analysis 1 (1966) 145-153.

[6] G.C. Sih and B. MacDonald, Engineering Fracture Mechanics 6 (1974) 361-386.

[7] G.C. Sih and E. Madenci, Engineering Fracture Mechanics 18 (1983) 667-677.

[8] A.R. Luxmoore, M.F. Light and W.T. Evans, International Journal of Fracture 13 (1977) RCR 257-259.

[9] M.F. Light and A.R. Luxmoore, Journal of Strain Analysis 12 (1977) 293-304.

[10] C.A. Griffis, Elastic-Plastic Fracture Toughness: A Comparison of J-integral and Crack Opening Displacement Characterizations, ASME Paper No. 75-PVP-38 (1975).

[11] A.D. Batte, W.S. Blackburn, A. Elsender, T.K. Hellen and A.D. Jackson, International Journal of Fracture 21 (1983) 49-66.

[12] G.C. Sih and B.V. Kiefer, in Nonlinear and Dynamic Fracture Mechanics Edited by N. Perrone and A.N. Atluri, Vol. 35, ASME Applied Mechanics Division (1979).

[13] G.C. Sih and E. Madenci, Engineering Fracture Mechanics 18 (1983) 1159-1171. 
[14] R. Hill, Journal of Mechanics and Physics of Solids 7 (1959) 209-225.

[15] Jilin Xu, Acta Mechanics Sinica 3 (1982) 272-279.

[16] R.M. McMeeking and J.R. Rice, International Journal of Solids and Structures 11 (1975) 601-616.

[17] C.L. Chow and Jilin Xu, Application of the Strain Energy Density Criterion to Ductile Fracture (in press).

[18] G.C. Sih, International Journal of Fracture 10 (1974) 305-321.

[19] Jilin Xu, Yinian Xue and Jinhu Han, in Procedings ICF International Symposium on Fracture Mechanics (1983) 267-274

[20] L.M. Kachanov, Foundation of the Theory of Plasticity, North-Holland Publishing, Amsterdam (1971).

\section{Appendix}

Let the stress and strain incremental deviator tensors be expressed as

$\sigma_{i j}^{\prime}=\sigma_{i j}-\sigma \delta_{i j}$

$\mathrm{d} \epsilon_{i j}^{\prime}=\mathrm{d} \epsilon_{i j}-\delta_{i j} \mathrm{~d} \epsilon$,

where

$\sigma=\frac{1}{3} \sigma_{\ell \ell}$

$\mathrm{d} \epsilon=\frac{1}{3} \mathrm{~d} \epsilon_{k k}$.

The strain energy density function is then

$$
\begin{aligned}
\frac{\mathrm{d} W}{\mathrm{~d} V} & =\int \sigma_{i j} \mathrm{~d} \epsilon_{i j}=\int\left(\sigma_{i j}^{\prime}+\sigma \delta_{i j}\right)\left(\mathrm{d} \epsilon_{i j}^{\prime}+\delta_{i j} \mathrm{~d} \epsilon\right)=\int\left[\sigma_{i j}^{\prime} \mathrm{d} \epsilon_{i j}^{\prime}+3 \sigma \mathrm{d} \epsilon\right] \\
& =\int\left[\sigma_{i j}^{\prime} \mathrm{d} \epsilon_{i j}^{\prime}+\frac{1}{3} \sigma_{\ell \ell} \mathrm{d} \epsilon_{k k}\right]=\int \sigma_{i j}^{\prime} \mathrm{d} \epsilon_{i j}^{\prime}+\frac{1}{3} \int \sigma_{\ell \ell} \mathrm{d} \epsilon_{k k} .
\end{aligned}
$$

The first and second terms are defined respectively by Kachanov [20] as the increment in the work of shape deformation and the increment in the elastic energy of volumetric compression. Similar first term was deduced by Hill [4] and known as work of distortion. Since the strain energy density function ( $\mathrm{d} W / \mathrm{d} V$ ) is composed of dilatation energy $(\mathrm{d} W / \mathrm{d} V)_{\mathrm{v}}$ and distortional energy $(\mathrm{d} W / \mathrm{d} V)_{\mathrm{d}}$, these two terms may be individually expressed as

$\left(\frac{\mathrm{d} W}{\mathrm{~d} V}\right)_{v}=\frac{1}{3} \int \sigma_{\ell \ell} \mathrm{d} \epsilon_{k k}$

and

$\left(\frac{\mathrm{d} W}{\mathrm{~d} V}\right)_{\mathrm{d}}=\int \sigma_{i j}^{\prime} \mathrm{d} \epsilon_{i j}^{\prime}$ 